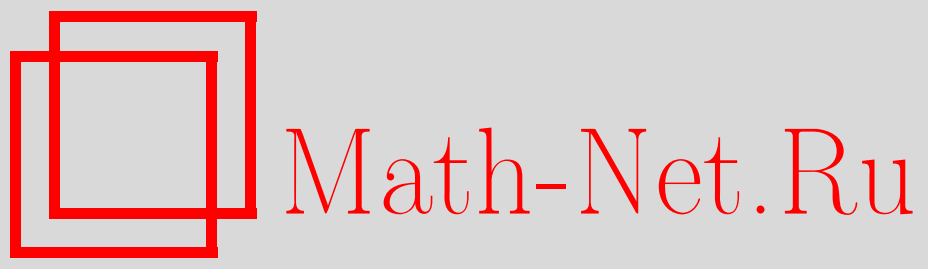

С. А. Кругляк, Л. А. Назарова, А. В. Ройтер, Ортоскалярные представления колчанов, соответствующих расширенным графам Дынкина, в категории гильбертовых пространств, Функи. анализ и его прил., 2010, том 44, выпуск 2, 57-73

DOI: https://doi.org/10.4213/faa2986

Использование Общероссийского математического портала MathNet.Ru подразумевает, что вы прочитали и согласны с пользовательским соглашением

http: //www . mathnet.ru/rus/agreement

Параметры загрузки:

IP : 18.234 .197 .8

26 апреля 2023 г., 09:02:40

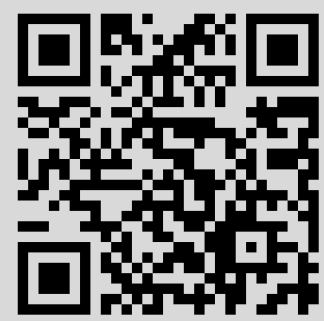




\title{
Ортоскалярные представления колчанов, соответствующих расширенным графам Дынкина, в категории гильбертовых пространств
}

\author{
(c) 2010. С. А. Кругляк, Л. А. НАзАровА, А. В. РойтеР
}

\begin{abstract}
Известно, что конечно представимые колчаны связаны с графами Дынкина, а ручные - с расширенными графами Дынкина. Ранее авторы распространили некоторые из этих результатов на локально скалярные (в дальнейшем названные ортоскалярными) представления колчанов в гильбертовых пространствах, в частности, доказали аналог теоремы Габриеля. В настоящей статье мы изучаем связь между неразложимыми представлениями в категории ортоскалярных представлений и неразложимыми представлениями в категории всех представлений колчана. Для колчанов, связанных с расширенными графами Дынкина, неразложимые ортоскалярные представления классифицируются с точностью до унитарной эквивалентности.
\end{abstract}

\section{Введение}

Более 30-ти лет назад (см. [1]) в работах И. М. Гельфанда, В. А. Пономарева, П. Габриеля и авторов было показано, что ряд проблем линейной алгебры (возникших в теории представлений алгебр, теории групп и модулей ХаришЧандры) допускают содержательное изучение как на наивном языке приведения наборов матриц теми или иными допустимыми преобразованиями, так и в категорно-функторных терминах. Отметим представления колчанов и частично упорядоченных множеств. Было доказано, в частности, что конечно представимые (ручные) колчаны соответствуют графам (расширенным графам) Дынкина.

В [2] был предложен метод перенесения этих результатов на представления колчанов в категории сепарабельных гильбертовых пространств (конечномерных (унитарных) и счетномерных), построены функторы Кокстера и доказан аналог теоремы Габриеля [3] для локально скалярных представлений колчанов (в дальнейшем мы заменим термин «локально скалярные представления» [2] на термин «ортоскалярные представления», считая его более удачным). У колчанов, соответствующих расширенным графам Дынкина, размерности неразложимых ортоскалярных представлений не ограничены в совокупности (см. [2]), но, как следует из [4]-[6], среди бесконечно представимых колчанов эти колчаны и только они не имеют бесконечномерных неразложимых ортоскалярных представлений.

В работе [7] начато изучение ортоскалярных представлений колчанов ручного типа, в частности, классифицируются все неразложимые представления колчанов с графом $\widetilde{D}_{4}$. В настоящей статье мы даем описание неразложимых представлений колчанов, соответствующих расширенным графам Дынкина $\widetilde{D}_{n}$, $\widetilde{E}_{6}, \widetilde{E}_{7}, \widetilde{E}_{8}, \widetilde{A}_{m}$ при четном $m$ (см. теоремы $\left.1-3\right)$. 


\section{$\S 1$. О неразложимости в категории представлений колчана и ее подкатегории ортоскалярных представлений}

Напомним некоторые определения и факты (см. [7]) об ортоскалярных представлениях колчанов. Колчан $Q$ с множеством вершин $Q_{v},\left|Q_{v}\right|=N$, и множеством стрелок $Q_{a}$ называется разделенным, если $Q_{v}=\stackrel{\circ}{Q} \sqcup \stackrel{\bullet}{Q}$ и для любой стрелки $\alpha \in Q_{a}$ ее начало $t_{\alpha}$ принадлежит $\stackrel{\circ}{Q}$, а конец $h_{\alpha}$ принадлежит $\dot{Q}$. Колчан $Q$ называется однократным, если при $\alpha \neq \beta$ либо $t_{\alpha} \neq t_{\beta}$, либо $h_{\alpha} \neq h_{\beta}$. Вершины из $\stackrel{\circ}{Q}$ будем называть четными, вершины из $\dot{Q}-$ нечетными.

Пусть $m=|\stackrel{\bullet}{Q}|, n=|\stackrel{\circ}{Q}|, \stackrel{\bullet}{Q}=\left\{i_{1}, \ldots, i_{m}\right\}, \stackrel{\circ}{Q}=\left\{j_{1}, \ldots, j_{n}\right\}$. Представление $T$ колчана $Q$ ставит в соответствие вершине $i \in Q_{v}$ конечномерное линейное пространство $T(i)$, а стрелке $\alpha: j \rightarrow i, \alpha \in Q_{a}$, линейное отображение $T_{i j}: T(j) \rightarrow T(i)$. Представление $T$ разделенного однократного колчана при фиксированных базисах пространств $T(i), i \in Q_{v}$, можно ассоциировать с блочной матрицей $T=\left[T_{i_{l}, j_{k}}\right]_{k=1, \ldots, n}^{l=1, \ldots, m}$, считая, что $T_{i_{l}, j_{k}}=0$, если не существует стрелки $\alpha \in Q_{a}$, такой, что $t_{\alpha}=j_{k}, h_{\alpha}=i_{l}$.

Пусть $\operatorname{Rep} Q-$ категория представлений колчана $Q$, объекты которой суть представления, а морфизм представления $T$ в представление $\widetilde{T}$ определяется как семейство линейных отображений $C=\left\{C_{i}\right\}_{i \in Q_{v}}, C_{i}: T(i) \rightarrow \widetilde{T}(i)$, таких, что для каждой стрелки $\alpha \in Q_{a}$ с $t_{\alpha}=j, h_{\alpha}=i$ диаграмма

$$
\begin{array}{cc}
T(j) \stackrel{T(\alpha)}{\longrightarrow} & T(i) \\
\downarrow C_{j} & \downarrow^{C_{i}} \\
\widetilde{T}(j) \stackrel{\widetilde{T}(\alpha)}{\longrightarrow} \widetilde{T}(i)
\end{array}
$$

коммутативна, т. е. $C_{i} T_{i j}=\widetilde{T}_{i j} C_{j}$.

Пусть представления $T, \widetilde{T}$ заданы матрицами

$$
T=\left[T_{i_{l}, j_{k}}\right]_{k=1, \ldots, n}^{l=1, \ldots, m}, \quad \widetilde{T}=\left[\widetilde{T}_{i_{l}, j_{k}}\right]_{k=1, \ldots, n}^{l=1, \ldots, m}
$$

и $A=\operatorname{diag}\left\{C_{i_{1}}, \ldots, C_{i_{m}}\right\}, B=\operatorname{diag}\left\{C_{j_{1}}, \ldots, C_{j_{n}}\right\}$. Тогда из коммутативности диаграмм (1) следует, что

$$
A T=\widetilde{T} B .
$$

Будем в дальнейшем также пользоваться обозначением $C=(A, B)$.

Пусть $\mathscr{H}$ - категория унитарных (конечномерных гильбертовых) пространств. Обозначим через $\operatorname{Rep}(Q, \mathscr{H})$ подкатегорию в $\operatorname{Rep} Q$, объекты которой являются представлениями $T$, такими, что $T(i), i \in Q_{v},-$ унитарные пространства, а морфизмы $C: T \rightarrow \widetilde{T}-$ это те из морфизмов в $\operatorname{Rep} Q$, для которых, кроме (1), коммутативными будут и диаграммы

$$
\begin{array}{cc}
T(j) & \stackrel{T(\alpha)^{*}}{\stackrel{T}{ }} T(i) \\
\downarrow C_{j} & \downarrow^{C_{i}} \\
\widetilde{T}(j) \stackrel{\widetilde{T}(\alpha)^{*}}{\longleftarrow} \widetilde{T}(i)
\end{array}
$$


т. е. будут выполняться равенства

$$
A T=\widetilde{T} B, \quad B T^{*}=\widetilde{T}^{*} A
$$

Представления $T, \widetilde{T}$ из $\operatorname{Rep} Q($ соответственно из $\operatorname{Rep}(Q, \mathscr{H}))$ эквивалентны в $\operatorname{Rep} Q($ соответственно в $\operatorname{Rep}(Q, \mathscr{H}))$, если найдется обратимый морфизм $C: T \rightarrow \widetilde{T}$. Можно показать, что $T$ и $\widetilde{T}$ эквивалентны в $\operatorname{Rep}(Q, \mathscr{H})$ тогда и только тогда, когда они унитарно эквивалентны (см., например, [8]), т. е. обратимый морфизм можно выбрать состоящим из унитарных матриц $C_{i}$.

Положим

$$
\overrightarrow{T_{i}}=\left[T_{i, j_{1}} ; \ldots ; T_{i, j_{n}}\right], \quad T_{j}^{\downarrow}=\left[\begin{array}{c}
T_{i_{1}, j} \\
\vdots \\
T_{i_{m}, j}
\end{array}\right] .
$$

Представление $T$ однократного разделенного колчана $Q$ из категории $\operatorname{Rep}(Q, \mathscr{H})$ назовем ортоскалярным, если каждой вершине $i \in Q_{v}$ сопоставлено действительное неотрицательное число $\chi(i)$ и выполняются следующие условия:

$$
\begin{gathered}
\vec{T}_{i} \cdot \vec{T}_{i}^{*}=\chi(i) I_{i} \quad \text { при } i \in \stackrel{\bullet}{Q}, \\
T_{j}^{\downarrow *} \cdot T_{j}^{\downarrow}=\chi(j) I_{j} \quad \text { при } j \in \stackrel{\circ}{Q} ;
\end{gathered}
$$

здесь $I_{i}$ - матрица единичного оператора в $T(i)$. Ортоскалярной будем называть и матрицу представления $T$. Набор $\chi=\{\chi(i)\}_{i \in Q_{v}}$ будем называть характером ортоскалярного представления $T$.

В определении представлений колчана $Q$ в $\mathscr{H}$ можно было бы отказаться от конечномерности пространств $T(i)$ и рассматривать также бесконечномерные представления, повторяя предыдущие определения почти дословно (ставя в соответствие вершине сепарабельное гильбертово пространство, а стрелке ограниченное линейное отображение). Поскольку все неразложимые ортоскалярные представления колчанов, соответствующих расширенным графам Дынкина, конечномерны, то для разложимых представлений в категории гильбертовых пространств есть общие теоремы (см. [9]) о разложении в прямую сумму (или прямой интеграл) неразложимых.

Будем говорить, что $Q$ (ортоскалярно) конечно представим в $\mathscr{H}$, если все его ортоскалярные представления распадаются в прямую сумму (конечную либо бесконечную) конечномерных представлений, размерности его неразложимых представлений ограничены в совокупности и в каждой размерности число неразложимых представлений с данным характером конечно. В противном случае будем называть $Q$ бесконечно представимым.

Определим категорию $\operatorname{Rep}_{\text {os }}(Q, \mathscr{H})$ как полную подкатегорию в $\operatorname{Rep}(Q, \mathscr{H})$, объекты которой суть ортоскалярные представления колчана $Q$.

Заметим, что предположение о разделенности колчана является чисто техническим. Пусть колчанам $Q$ и $Q^{\prime}$ соответствует один и тот же граф $G$, но ориентации разные. Пусть $X(a)=\left\{\alpha \in Q_{a} \mid t_{\alpha}=a\right\}, Y(a)=\left\{\alpha \in Q_{a} \mid h_{\alpha}=a\right\}$, $a \in Q_{v}$. Представление $T$ колчана $Q$ является ортоскалярным с характером $\chi=\{\chi(a)\}_{a \in Q_{v}}$, если для любого $a \in Q_{v}$

$$
\sum_{\alpha \in X(a)} T^{*}(\alpha) T(\alpha)+\sum_{\beta \in Y(a)} T(\beta) T^{*}(\beta)=\chi(a) I_{T(a)} .
$$


Морфизмы между представлениями определяются коммутативными диаграммами $(1),(3)$. В этом случае определяется категория $\operatorname{Rep}_{\text {os }}(Q, \mathscr{H})$. Легко видеть что между объектами и морфизмами категорий $\operatorname{Rep}_{\text {os }}(Q, \mathscr{H})$ и $\operatorname{Rep}_{\text {os }}\left(Q^{\prime}, \mathscr{H}\right)$ естественным образом устанавливается взаимно однозначное соответствие и категории $\operatorname{Rep}_{\text {os }}(Q, \mathscr{H})$ и $\operatorname{Rep}_{\text {os }}\left(Q^{\prime}, \mathscr{H}\right)$ эквивалентны. В этом смысле ортоскалярные представления колчанов не зависят от выбора ориентации ребер, что оправдывает выбор технически удобной ориентации (разделенные колчаны).

Пусть $G$ - граф и $G_{v}$ - множество его вершин. Обозначим через $\mathbb{R}^{G_{v}}$ линейное действительное пространство, состоящее из наборов $x=\left(x_{i}\right)$ действительных чисел $x_{i}\left(i \in G_{v}\right)$; элементы из $\mathbb{R}^{G_{v}}$ будем называть $G$-векторами. $G$-вектор $x$ будем называть положительным $(x>0)$, если $x \neq 0$ и $x_{i} \geqslant 0$ для $i \in G_{v}$. Положим $\mathbb{R}_{+}^{G_{v}}=\left\{x \in \mathbb{R}^{G_{v}} \mid x>0\right\}$.

Пусть $T$ - ортоскалярное представление разделенного однократного колчана $Q$ и $G$ - соответствующий этому колчану граф. Сопоставим представлению $T$ два $N$-мерных $G$-вектора $(N=m+n)$ : размерность $d=\{d(j)\}_{j \in Q_{v}}$, где $d(j)=\operatorname{dim} T(j)$, и характер $\chi=\{\chi(j)\}_{j \in Q_{v}}$, определенный выше (см. (5), (6)). Два ортоскалярных представления $T$ и $\widetilde{T}$ эквивалентны, если существуют такие унитарные матрицы $U=\operatorname{diag}\left\{U_{i_{1}}, \ldots, U_{i_{m}}\right\}$ и $V=\operatorname{diag}\left\{V_{j_{1}}, \ldots, V_{j_{n}}\right\}$, что

$$
U T=\widetilde{T} V, \quad \text { или } \quad \widetilde{T}_{i_{l}, j_{k}}=U_{i_{l}} T_{i_{l}, j_{k}} V_{j_{k}}^{*} .
$$

Представление $T$ будем называть шуровским (brick) в категории $\operatorname{Rep} Q$ (coответственно $\left.\operatorname{Rep}(Q, \mathscr{H}), \operatorname{Rep}_{\text {os }}(Q, \mathscr{H})\right)$, если его кольцо эндоморфизмов в этой категории одномерно (изоморфно $\mathbb{C}$ ). Очевидно, что если $T$ - шуровское представление, то оно неразложимо (в соответствующей категории). Как известно, если $T$ неразложимо в категории $\operatorname{Rep}(Q, \mathscr{H})$, то оно в ней шуровское (см., например, [7, замечание 4 и его доказательство]).

Представление $T$ колчана $Q$ называется точным, если $T(i) \neq 0$ при всех $i \in Q_{v}$. Носителем представления $T$ называется множество $Q_{v}^{T}=\left\{i \in Q_{v} \mid\right.$ $T(i) \neq 0\}$. Характер ортоскалярного представления $T$ определен однозначно на его носителе $Q_{v}^{T}$ (и неоднозначно вне носителя). Если $Q_{v}^{T}=Q_{v}$, то характер определен однозначно и обозначается через $\chi_{T}$. В общем случае обозначим через $\left\{\chi_{T}\right\}$ множество всех характеров представления $T$. Ясно, что если $T$ и $\widetilde{T}$ унитарно эквивалентны, то $\left\{\chi_{T}\right\}=\left\{\chi_{\widetilde{T}}\right\}$.

Теорема 1. Пусть $Q-$ разделенный однократный колчан.

(a) Eсли $T, \widetilde{T} \in \operatorname{Rep}_{\text {ов }}(Q, \mathscr{H})$ - представления с одинаковыми характерами и $T$ эквивалентно $\widetilde{T}$ в $\operatorname{Rep} Q$, то $T$ эквивалентно $\widetilde{T}$ в $\operatorname{Rep}_{\text {os }}(Q, \mathscr{H})$.

(b) Если $T$ - неразложимое представление в $\operatorname{Rep}_{\text {os }}(Q, \mathscr{H})$, то оно является неразложимым и, более того, шуровским и в $\operatorname{Rep} Q$.

Для доказательства этой теоремы нужны следующие 3 леммы.

Лемма 1. Пусть $C$-морфизм представления $T$ в $\widetilde{T}$ в $\operatorname{Rep} Q$, m.е. $A T=$ $\widetilde{T} B$ и $A, B$ - унитарные отображения. Тогда $C$ является морфизмом представления $T$ в представление $\widetilde{T}$ и в $\operatorname{Rep}_{\text {os }}(Q, \mathscr{H})$, m.е. $B T^{*}=\widetilde{T}^{*} A$.

Доказательство. Из (2) следует, что $T^{*} A^{*}=B^{*} \widetilde{T}^{*}$ или, учитывая унитарность отображений $A$ и $B, T^{*} A^{-1}=B^{-1} \widetilde{T}^{*}$. Поэтому $B T^{*}=\widetilde{T}^{*} A$. 
Лемма 2. Пусть $C$-эндоморфизм представления $T$ в $\operatorname{Rep} Q$, m.e. $A T=$ TB и $A, B$ - самосопрлжсенные операторы. Тогда $C$ есть эндоморфизм представления $T$ и в $\operatorname{Rep}(Q, \mathscr{H})$, т.е. $T^{*} A=B T^{*}$.

Доказательство. Равенство $B T^{*}=T^{*} A$ получается из равенства $A T=T B$ сопряжением.

Если $\vec{x}=\left(x_{1}, \ldots, x_{n}\right), x_{i} \in \mathbb{C}$, то длиной строки $\vec{x}$ назовем число $|\vec{x}|=$ $\sqrt{\sum_{i=1}^{n} x_{i} \bar{x}_{i}}$. Аналогично определяется длина $\left|y^{\downarrow}\right|$ для столбца.

Лемма 3. Пусть $Z=\left[z_{i j}\right]_{i=1, \ldots, m}^{j=1, \ldots, n}, W=\left[w_{i j}\right]_{i=1, \ldots, m}^{j=1, \ldots, n}-$ матрицы над полем $\mathbb{C}$, имеющие одинаковые положительные длины $|\vec{x}|,|y \downarrow|$ соответствующих строк и столбиов. Пусть $A=\operatorname{diag}\left\{a_{1}, \ldots, a_{m}\right\}, B=\operatorname{diag}\left\{b_{1}, \ldots, b_{n}\right\}, a_{i}>0$, $b_{j}>0$, и пусть $A Z=W B$. Тогда $Z=W$.

Формулировка и доказательство леммы 3 содержатся в [7, лемма 1].

Доказательство теоремы 1. (а) Пусть $C=(A, B)$ осуществляет эквивалентность представлений $T, \widetilde{T}$ из $\operatorname{Rep}_{\text {os }}(Q, \mathscr{H})$ в категории $\operatorname{Rep} Q$, т. е. $A T=$ $\widetilde{T} B$ ( $A$ и $B$ - обратимые матрицы). Пусть $A=X U, B=V Y$ - полярные разложения матриц $A$ и $B$, где $U, V$ - унитарные, а $X, Y$ - положительные невырожденные матрицы (при этом можно считать, что $U, V, X, Y$ имеют такую же блочно-диагональную структуру, как $A$ и $B$ ). Пусть, кроме того, $X=U_{1}^{*} \widetilde{X} U_{1}, Y=V_{1} \widetilde{Y} V_{1}^{*}$, где $U_{1}, V_{1}$ - унитарные матрицы, а $\widetilde{X}$, $\widetilde{Y}$ - диагональные матрицы с положительными числами на диагонали. Тогда $U_{1}^{*} \widetilde{X} U_{1} U T=\widetilde{T} V V_{1} \widetilde{Y} V_{1}^{*}$, или $\widetilde{X}\left(U_{1} U T V_{1}\right)=\left(U_{1} \widetilde{T} V V_{1}\right) \widetilde{Y}$. Из предположения, что $T$ и $\widetilde{T}$ являются ортоскалярными представлениями с одинаковыми характерами, следует, что $\vec{T}_{i} \vec{T}_{i}^{*}=\overrightarrow{\widetilde{T}}_{i} \overrightarrow{\widetilde{T}}_{i}^{*}=\chi(i) I_{i}$, а это, в частности, означает, что длины соответствующих строк матриц $\vec{T}_{i}$ и $\overrightarrow{\widetilde{T}}_{i}$, а значит, и $T$ и $\widetilde{T}$ равны (аналогичное утверждение верно и для столбцов). При допустимых унитарных преобразованиях матриц $T$ и $\widetilde{T}$ (при переходе к матрицам $U_{1} U T V_{1}$ и $U_{1} \widetilde{T} V V_{1}$ ) их ортоскалярность сохраняется и характеры не меняются, так что длины соответствующих строк и столбцов матриц $U_{1} U T V_{1}$ и $U_{1} \widetilde{T} V V_{1}$ равны и потому $U_{1} U T V_{1}=U_{1} \widetilde{T} V V_{1}$ (лемма 3$)$, а тогда, после сокращений, получаем $U T=\widetilde{T} V$, и $T$ и $\widetilde{T}$ эквивалентны в $\operatorname{Rep}_{\text {os }}(Q, \mathscr{H})$ по лемме 1.

Пункт (b) доказан в [7, теорема 1].

\section{§2. Размерности неразложимых ортоскалярных представлений}

С колчаном $Q$ связана форма Tитса $q(x)$ на $\mathbb{R}^{Q_{v}}$ :

$$
q(x)=\sum_{i \in Q_{v}} x_{i}^{2}-\sum_{\alpha \in Q_{a}} x_{t_{\alpha}} x_{h_{\alpha}}, \quad x \in \mathbb{R}^{Q_{v}} .
$$

Из [10] следует, что размерности неразложимых в $\operatorname{Rep} Q$ представлений для графов и расширенных графов Дынкина совпадают с решениями уравнений $q(x)=1$ (действительные корни) и $q(x)=0$ (мнимые корни), $x \in \mathbb{Z}_{+}^{Q_{v}}=\{x \in$ $\left.\mathbb{Z}^{Q_{v}} \mid x \neq 0, x_{i} \geqslant 0\right\}$. Мнимые корни кратны минимальному мнимому положительному корню $\delta=\delta_{G}$. 
Фиксируем нумерацию вершин в $\stackrel{\bullet}{Q}=\left\{i_{1}, \ldots, i_{m}\right\}$ и $\stackrel{\circ}{Q}=\left\{j_{1}, \ldots, j_{n}\right\}$ (для определенности будем считать, что узловая точка, если она одна, лежит в $\dot{Q}$; будем вершины из $\stackrel{\circ}{Q}$ обозначать также символами $\left.i_{m+k}=j_{k}, k=i, \ldots, n\right)$. Пусть $x \in \mathbb{R}^{Q_{v}}, x_{k}=x\left(i_{k}\right)$, где $k=1, \ldots, m+n, c$ - преобразование Кокстера на $\mathbb{R}^{Q_{v}}, c=\sigma_{i_{n+m}} \cdots \sigma_{i_{2}} \sigma_{i_{1}},\left(\sigma_{i_{k}}(x)\right)_{k}=-x_{k}+\sum_{l, i_{l}-i_{k}} x_{l},\left(\sigma_{i_{k}}(x)\right)_{l}=x_{l}$ при $l \neq k$. Ясно, что $\sigma_{i}^{2}=\mathrm{id}$ при $i=1, \ldots, n+m$. Поэтому $c^{-1}=\sigma_{i_{1}} \cdots \sigma_{i_{n+m}}$. Будем также пользоваться обозначениями $\stackrel{\bullet}{c}=\sigma_{i_{n}} \cdots \sigma_{i_{1}}, \stackrel{\circ}{c}=\sigma_{i_{m+n}} \cdots \sigma_{i_{m+1}}$ и называть эти преобразования отражениями Кокстера $\left(\stackrel{\bullet}{c}^{2}=\mathrm{id}, \stackrel{\circ}{c}^{2}=\mathrm{id}\right)$. Положим $\dot{\bullet}_{k}=\underbrace{\ldots . \dot{c} c \bullet}_{k \text { раз }}$, $\stackrel{\circ}{c}_{k}=\underbrace{\ldots \stackrel{\circ}{c} c \stackrel{\circ}{*}}_{k \text { раз }}, k \in \mathbb{N}$. Вектор $x \in \mathbb{R}_{+}^{G_{v}}$ регулярен, если $c^{t}(x) \in \mathbb{R}_{+}^{G_{v}}$ при любом $t \in \mathbb{Z}$, и сингулярен в противном случае (терминология восходит к [11]).

Представление $T$ колчана $Q$ сингулярно, если оно неразложимо, конечномерно и его размерность $d-$ сингулярный вектор; $T$ регулярно, если $T$ неразложимо, конечномерно и не сингулярно.

Пусть $\delta_{G}=\left(\delta_{1}, \ldots, \delta_{m}, \delta_{m+1}, \ldots, \delta_{m+n}\right)$. Построим линейную форму

$$
L_{G}(x)=\sum_{i_{k} \in \dot{G}} \delta_{k} x_{k}-\sum_{i_{m+k} \in \dot{G}} \delta_{m+k} x_{m+k}, \quad x \in \mathbb{R}^{G_{v}} .
$$

Справедливо следующее утверждение (см., например, [12]): пусть $G$ - расширенный граф Дынкина; для того чтобы корень $x \in \mathbb{R}^{G_{v}}$ был сингулярным, необходимо и достаточно, чтобы $L_{G}(x) \neq 0$. Отметим, что у графа Дынкина все корни действительны и сингулярны, у расширенного графа Дынкина все мнимые корни регулярны, а действительные корни разбиваются на сингулярные и регулярные. В [13] доказана следующая лемма.

Лемма 4. Если $T$ - неразложимое в $\operatorname{Rep} Q$ регулярное представление колчана $Q$, граф $G$ которого есть расширенный граф Дынкина, то $T$ является шуровским представлением тогда и только тогда когда $\operatorname{dim} T=d \leqslant \delta_{G}$ при покоординатном сравнении векторов.

Докажем следующее утверждение:

Лемма 5. Пусть $G$ - расширенный граф Дынкина, а $d \in \mathbb{Z}_{+}^{G}-$ корень графа $G$, удовлетворяющий условию $d<\sigma_{G}$. Тогда $d$ либо является неточным корнем (одна из координат нулевая), либо получается из некоторого неточного корня $\tilde{d}$ применением отражений Кокстера $\stackrel{\bullet}{c}$ и $\stackrel{\circ}{c}$.

Доказательство. Достаточно показать, что такой точный корень можно отражениями Кокстера преобразовать в неточный корень $\widetilde{d}$.

Рассмотрим граф $\widetilde{D}_{n}$

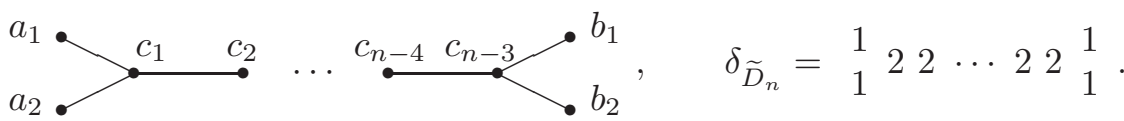


Пусть $d=\left(d_{a_{1}}, d_{a_{2}}, d_{c_{1}}, \ldots, d_{c_{n-3}}, d_{b_{1}}, d_{b_{2}}\right)$ (координаты вектора $\delta_{\widetilde{D}_{n}}$ располагаются так же, как соответствующие вершины в графе). Так как $d$ - точный корень, то $d_{a_{1}}=d_{a_{2}}=d_{b_{1}}=d_{b_{2}}=1$. Назовем отмеченнымм первый индекс $k$, для которого $x_{c_{k}}=1$ (т. е. $\left.x_{c_{1}}=x_{c_{2}}=\cdots=x_{c_{k-1}}=2\right)$ ). Пусть для определенности $c_{k-1} \in \dot{\bullet}$. Тогда у $\stackrel{\bullet}{c} d$ координаты $x_{a_{1}}, x_{a_{2}}, x_{c_{1}}, \ldots, x_{c_{k-2}}$ совпадают с соответствующими координатами вектора $d$, а $x_{c_{k-1}}=1$, т. е. отмеченный индекс уменьшается на единицу. Применяя к $\stackrel{\bullet}{c}$ преобразование $\stackrel{\circ}{c}$, продолжим этот процесс. В результате мы придем к вектору, у которого отмеченный индекс равен 1, а стало быть, на следующем шаге получим вектор, у которого $x_{a_{1}}=x_{a_{2}}=0$. Это и будет искомый неточный вектор $\tilde{d}$.

Для графов $\widetilde{E}_{6}, \widetilde{E}_{7}, \widetilde{E}_{8}$ результат можно получить аналогично или прямым перебором (положительных корней $d$, таких, что $d<\delta_{G}$, конечное число).

Далее, $\delta_{\widetilde{A}_{n}}=(1, \ldots, 1)$, так что из условия $d<\delta_{\widetilde{A}_{n}}$ следует, что одна из координат нулевая и $d$ есть неточный вектор.

\section{§3. Функторы Кокстера и ортоскалярные представления, размерности которых суть действительные корни}

Пусть $g \in Q_{v}$ и $\Pi_{g}$ - простейшее представление колчана $Q: \Pi_{g}(g)=\mathbb{C}$, $\Pi_{g}(i)=0$ при $i \neq g, i \in Q_{v}$. Ясно, что если $f_{g}$ - характер представления $\Pi_{g}$, то $f_{g}(g)=0$; будем предполагать, что $f_{g}(i)>0$ при $i \neq g$.

Обозначим через $\operatorname{Rep}(Q, d, \chi)$ категорию, объектами которой являются пары $(T, \chi)$, где $T$ - представление из $\operatorname{Rep}_{\mathrm{os}}(Q, \mathscr{H})$ размерности $d$, а $\chi$ - его фиксированный характер (значения которого однозначно определяются по $T$ лишь на носителе $Q_{v}^{T}$ представления $\left.T\right)$. Так как $\chi(i) I_{i}=\vec{T}_{i} \vec{T}_{i}{ }^{*}$ либо $\chi(i) I_{i}=T_{i}{ }^{\downarrow} T_{i}{ }^{\downarrow}$, то $\chi(i) \geqslant 0$. Будем предполагать, что вне $Q_{v}^{T}$ характер $\chi$ может принимать любые положительные значения. В этом смысле объекты $(T, \chi)$ при фиксированном $T$ зависят от $\left|Q_{v}\right|-\left|Q_{v}^{T}\right|$ положительных параметров. Морфизмы объекта $(T, \chi)$ в объект $(\widetilde{T}, \chi)$ отождествляются с морфизмами представления $T$ в $\widetilde{T}$ в категории $\operatorname{Rep}_{\text {os }}(Q, \mathscr{H})$.

Если $(T, \chi) \in \operatorname{Rep}(Q, d, \chi)$ и $\chi\left(i_{0}\right)=0, d\left(i_{0}\right) \neq 0$, то либо $T$ - простейшее представление $\Pi_{i_{0}}$, локализованное в вершине $i_{0}$, либо $\Pi_{i_{0}}$ входит в $T$ прямым слагаемым. В предположении, что $T$ - неразложимое представление, не являющееся простейшим, из $d\left(i_{0}\right) \neq 0$ следует, что $\chi\left(i_{0}\right)>0$.

Пусть

$$
\begin{aligned}
& \stackrel{\circ}{Q}_{v}^{T}=\stackrel{\circ}{Q} \cap Q_{v}^{T}, \quad \stackrel{\bullet}{Q}_{v}^{T}=\stackrel{\bullet}{Q} \cap Q_{v}^{T}, \\
& S=\left\{(d, \chi) \in \mathbb{Z}_{+}^{Q_{v}} \times \mathbb{R}_{+}^{Q_{v}} \mid d(a)+\chi(a)>0 \text { для } a \in Q_{v}\right\}, \\
& S_{\circ}=\left\{(d, \chi) \in S \mid \chi(a)>0 \text { при } a \in \stackrel{\circ}{Q}_{v}^{T}\right\}, \\
& S_{\bullet}=\left\{(d, \chi) \in S \mid \chi(a)>0 \text { при } a \in \dot{Q}_{v}^{T}\right\} \text {, }
\end{aligned}
$$

$\operatorname{Rep}_{\circ}(Q, d, \chi) \subset \operatorname{Rep}(Q, d, \chi)($ соответственно $\operatorname{Rep}(Q, d, \chi) \subset \operatorname{Rep}(Q, d, \chi))-$ полная подкатегория, объектами которой являются пары $(T, \chi)$ с $(d, \chi) \in S_{\text {。 }}$ $\left(\right.$ соответственно $\left.(d, \chi) \in S_{\bullet}\right)$. 
В [2] были построены функторы отражсений Кокстера $\stackrel{\circ}{F}_{d, \chi}, \stackrel{\bullet}{F}_{d, \chi}, \stackrel{\circ}{F}, \stackrel{\bullet}{F}$ : $\stackrel{\circ}{F}_{d, \chi}: \operatorname{Rep}_{\circ}(Q, d, \chi) \rightarrow \operatorname{Rep}_{\circ}(Q, \stackrel{\circ}{c}(d), \stackrel{\circ}{\chi}), \quad \dot{\circ}_{d, \chi}: \operatorname{Rep}_{\bullet}(Q, d, \chi) \rightarrow \operatorname{Rep}(Q, \dot{c}(d), \dot{\chi})$, где

$$
\begin{aligned}
& \stackrel{\circ}{c}(d)(i)=\left\{\begin{array}{ll}
-d(i)+\sum_{j: j-i} d(j), & i \in \stackrel{\circ}{Q}, \\
d(i), & i \in \dot{\circ},
\end{array} \quad \cdot \quad(d)(i)= \begin{cases}-d(i)+\sum_{j: j-i} d(j), & i \in \dot{Q}, \\
d(i), & i \in \stackrel{\circ}{Q},\end{cases} \right. \\
& \stackrel{\circ}{\chi}(i)=\left\{\begin{array}{ll}
-\chi(i)+\sum_{j: j-i} \chi(j), & i \in \dot{\leftrightarrow}_{v}^{T}, \\
\chi(i), & i \notin \dot{\bullet}_{v}^{T},
\end{array} \quad \dot{\chi}(i)= \begin{cases}-\chi(i)+\sum_{j: j-i} \chi(j), & i \in \stackrel{\circ}{Q}_{v}^{T}, \\
\chi(i), & i \notin \stackrel{\circ}{Q}_{v}^{T} .\end{cases} \right.
\end{aligned}
$$

Определим (см. [2]) категорию $\operatorname{Rep}(Q, \amalg)$ следующим образом: $\operatorname{Ob} \operatorname{Rep}(Q, \amalg)$ $=\coprod_{(d, \chi) \in S} \operatorname{Ob} \operatorname{Rep}(Q, d, \chi)$, а морфизмы между объектами из $\operatorname{Ob} \operatorname{Rep}\left(Q, d_{1}, \chi_{1}\right)$ и $\operatorname{Ob} \operatorname{Rep}\left(Q, d_{2}, \chi_{2}\right)$ совпадают с морфизмами в $\operatorname{Rep}\left(Q, d_{1}, \chi_{1}\right)$ при $\left(d_{1}, \chi_{1}\right)=$ $\left(d_{2}, \chi_{2}\right)$ и отсутствуют при $\left(d_{1}, \chi_{1}\right) \neq\left(d_{2}, \chi_{2}\right)$. Определим полные подкатегории $\operatorname{Rep}_{\circ}(Q, \amalg)$ и $\operatorname{Rep}(Q, \amalg)$ в $\operatorname{Rep}(Q, \amalg)$. Множество объектов категории $\operatorname{Rep}_{\circ}(Q, \amalg)$ есть $\coprod_{(d, \chi) \in S_{\circ}} \operatorname{Ob} \operatorname{Rep}(Q, d, \chi)$, а множество объектов категории $\operatorname{Rep}(Q, \amalg)$ есть $\coprod_{(d, \chi) \in S} . \operatorname{Ob} \operatorname{Rep}(Q, d, \chi)$. Функторы $\stackrel{\circ}{F}_{d, \chi}\left(\dot{F}_{d, \chi}\right)$ естественным образом порождают функторы $\stackrel{\circ}{F}(\dot{F})$ четных (нечетных) отражений Кокстера,

$$
\stackrel{\circ}{F}: \operatorname{Rep}_{\circ}(Q, \amalg) \rightarrow \operatorname{Rep}_{\circ}(Q, \amalg), \quad \dot{F}: \operatorname{Rep}_{\bullet}(Q, \amalg) \rightarrow \operatorname{Rep}_{\bullet}(Q, \amalg),
$$

осуществляющих эквивалентность каждой из категорий в себя.

$$
\text { Положим } \stackrel{\circ}{F}_{k}=\underbrace{\ldots \stackrel{\circ}{*} \dot{\bullet} \stackrel{\circ}{F}}_{k \text { раз }}, \stackrel{\circ}{F}_{k}=\underbrace{\ldots \stackrel{\circ}{F} \stackrel{\circ}{F} \dot{\bullet}}_{k \text { раз }} \text {. }
$$

Замечание 1. В [2] функторы отражений Кокстера вводятся в предположении, что граф $G$ колчана $Q$ не содержит циклов. Конструкция функторов $\stackrel{\bullet}{F}, \stackrel{\circ}{F}$ практически без изменений переносится на все разделенные однократные колчаны, и для них имеют место формулы (8), (9).

Пусть $Q_{v}^{d}=\left\{i \in Q_{v} \mid d(i) \neq 0\right\}, Q^{d}$ - полный подколчан в $Q$ с множеством вершин $Q_{v}^{d}, \widehat{d}=\{d(i)\}_{i \in Q_{v}^{d}}$. Пусть Ind $Q^{d}-$ множество классов $[T]$ точных неразложимых ортоскалярных унитарно эквивалентных представлений $T$ колчана $Q^{d}$ размерности $\widehat{d}$ в $\operatorname{Rep}_{\text {os }}\left(Q^{d}, \mathscr{H}\right)$, a $\operatorname{Ind}(Q, d)$ - множество классов $[T, \chi]$ точных неразложимых эквивалентных объектов размерности $d$ в категории $\operatorname{Rep}(Q, \amalg)$. Если $d-$ точный вектор, то $Q^{d}=Q$ и множества $\operatorname{Ind}(Q, d)$ и Ind $Q$ естественным образом отождествляются. Если $(T, \chi) \in \operatorname{Rep}(Q, d, \chi)$, то, отождествляя класс $[T, \chi]$ с парой $\left([T],(\chi(a))_{a \in Q_{v} \backslash Q_{v}^{d}}\right)$, получаем, что $\operatorname{Ind}(Q, d)=$ Ind $Q^{d} \times \mathbb{R}_{>0}^{Q_{v} \backslash Q_{v}^{d}}$.

Пусть $Q$ - разделенный однократный колчан, граф $G$ которого есть расширенный граф Дынкина, $T$ - его точное неразложимое ортоскалярное представление размерности $d=(d(i))_{i \in Q_{v}}(d(i)>0)$ с характером $\chi=(\chi(i))_{i \in Q_{v}}$. 
Предположим, что $d$ - действительный корень графа $G$. Рассмотрим 2 случая: $d$ есть либо сингулярный корень, либо регулярный.

1) Пусть $d$ - сингулярный действительный корень графа $G$; тогда $d$ получается из некоторого простейшего корня $d_{g}\left(d_{g}(a)=0, a \neq g, d_{g}(g)=1\right)$ отражениями Кокстера. Если предположить для определенности, что $g \in \dot{G}$, то $d=\stackrel{\circ}{c}_{k} d_{g}$ для некоторого $k \in \mathbb{N}$ и для некоторого фиксированного характера $f_{g}(i)$ представления $\Pi_{g}$ функтор $\stackrel{\circ}{F}_{k}: \operatorname{Rep}_{\circ}\left(Q, d_{g}, f_{g}\right) \rightarrow \operatorname{Rep}_{\circ}(Q, d, \chi)$ переводит объект $\left(\Pi_{g}, f_{g}\right)$ в объект $(T, \chi)$. Так как $\stackrel{\circ}{F}_{k}$ - функтор эквивалентности категорий $\operatorname{Rep}_{\circ}\left(Q, d_{g}, f_{g}\right)$ и $\operatorname{Rep}_{\circ}(Q, d, \chi)$, то этим определяется взаимно однозначное отображение

$$
\phi: \mathbb{R}_{>0}^{Q_{v} \backslash\{0\}} \longrightarrow \operatorname{Ind}(Q, d)=\operatorname{Ind} Q,
$$

и в этом смысле точные неразложимые ортоскалярные представления размерности $d$ (определенные с точностью до унитарной эквивалентности) зависят от $\left|Q_{v}\right|-1$ положительных параметров.

2) Пусть $d$ - регулярный действительный корень графа $G$, являющийся размерностью неразложимого точного ортоскалярного представления $T$ колчана $Q$. Тогда $d$ получается из неточного корня $d^{\prime}$ отражениями Кокстера $\stackrel{\circ}{c}$ и $\dot{c}$ (см. леммы 4,5$)$. Пусть для определенности $d=\stackrel{\circ}{c}_{k} d^{\prime}$ и $k$ нечетное. Тогда $(T, \chi)$ получается из некоторого неточного неразложимого объекта $\left(T^{\prime}, \chi^{\prime}\right)$ с помощью функторов отражений Кокстера $\stackrel{\circ}{F}$ и $\stackrel{\bullet}{F}\left(d^{\prime}=\dot{c}_{k} d\right.$ и $\left.\left(T^{\prime}, \chi^{\prime}\right)=\dot{\bullet}_{k}(T, \chi)\right)$. $T^{\prime}$ есть точное представление колчана $Q^{d^{\prime}}$ размерности $\widehat{d}^{\prime}=\left\{d^{\prime}(i)\right\}_{i \in Q_{v}^{d^{\prime}}}$. Вектор $\widehat{d}^{\prime}$ есть сингулярный корень графа $G^{d^{\prime}}$, соответствующего колчану $Q^{d^{\prime}}$ (так как $G^{d^{\prime}}$ есть граф Дынкина - все его корни сингулярны). Следуя изложенному в п. 1), получаем, что функторы Кокстера в категории $\operatorname{Rep}\left(Q^{d^{\prime}}, \amalg\right)$ определяют взаимно однозначное отображение

$$
\phi_{1}: \mathbb{R}_{>0}^{Q_{v}^{d^{\prime}} \backslash\{0\}} \longrightarrow \operatorname{Ind}\left(Q^{d^{\prime}}, d^{\prime}\right)=\operatorname{Ind} Q^{d^{\prime}},
$$

а функторы Кокстера в категории $\operatorname{Rep}(Q, \amalg)$ - взаимно однозначное отображение

$$
\psi: \text { Ind } Q^{d^{\prime}} \times \mathbb{R}_{>0}^{Q_{v} \backslash Q_{v}^{d^{\prime}}} \longrightarrow \operatorname{Ind}(Q, d) .
$$

В результате получаем взаимно однозначное отображение

$$
\phi: \mathbb{R}_{>0}^{Q_{v}^{d^{\prime}} \backslash\{0\}} \times \mathbb{R}_{>0}^{Q_{v} \backslash Q_{v}^{d^{\prime}}} \longrightarrow \operatorname{Ind}(Q, d)=\operatorname{Ind} Q,
$$

и в этом смысле точные неразложимые ортоскалярные представления размерности $d$ (определенные с точностью до унитарной эквивалентности) зависят от $\left|Q_{v}\right|-1$ положительных параметров.

Таким образом, нами доказана следующая теорема.

Теорема 2. Пусть $Q-$ разделенный однократный колчан, граф $G$ которого есть расширенный граф Дынкина, а $d$ - его точный действительный корень. Тогда построенное с помощъю функторов отражений Кокстера $\stackrel{\circ}{F}$ и $\stackrel{\bullet}{F}$ отображение

$$
\phi: \mathbb{R}_{>0}^{Q_{v} \backslash\{0\}} \longrightarrow \operatorname{Ind}(Q, d)
$$


есть биекиия, и в этом смысле точные неразложимые ортоскалярные представления размерности $d$ колчана $Q$ зависят от $\left|Q_{v}\right|-1$ положительных параметров.

Заметим, что в доказательстве теоремы 2 фактически содержится алгоритм получения таких представлений из простейших с помощью функторов Кокстеpa.

\section{§4. Ортоскалярные представления, размерности которых суть мнимые корни}

Пусть $Q$ - разделенный однократный колчан, граф $G$ которого есть расширенный граф Дынкина. Из теоремы 1 и леммы 4 следует, что единственный мнимый корень, который может быть размерностью неразложимого ортоскалярного представления колчана $Q,-$ это $\delta_{G}$.

Докажем теорему аналогичную теореме 2 , но относящуюся к мнимым корням графа $G$ (заметим, что в случае $\chi_{T}=\delta_{G}$ для графов $\widetilde{D}_{4}, \widetilde{E}_{6}, \widetilde{E}_{7}$ на другом языке и другими методами унитарная классификация неразложимых представлений получена в [14]-[16]).

Теорема 3. Если $Q$ - разделенный однократный колчан, граф $G$ которого есть расширенный граф Дынкина, то в размерности $\delta_{G}$ неразложимые ортоскалярные представления колчана $Q$ зависят от $\left|Q_{v}\right|+1$ положительных параметров.

Теорему докажем фактическим описанием всех неразложимых представлений указанных колчанов в размерностях $\delta_{G}$.

а) Пусть $G=\widetilde{A}_{n}$ - цикл с $n$ вершинами. Из предположения о разделенности и однократности колчана следует, что $n$ четное и $n \geqslant 4$. Известно, что $\delta_{\widetilde{A}_{n}}=(1, \ldots, 1)$. Представление $T$ в размерности $\delta_{\widetilde{A}_{n}}$ задается $n$ ненулевыми комплексными числами. Переходом к унитарно эквивалентному представлению $n-1$ чисел можно сделать положительными $\left(\left|Q_{v}\right|-1\right.$ параметров); допустимые преобразования, не меняющие значения этих параметров, не меняют и $n$-е число (зависящее от двух положительных параметров - аргумента и модуля). Таким образом, $T$ зависит от $n$ параметров, принимающих произвольные положительные значения, и одного параметра $\phi$, принимающего значения на отрезке $(0,2 \pi]$, т. е. от $\left|Q_{v}\right|+1=n+1$ параметров.

b) Пусть $G=\widetilde{D}_{n}, n \geqslant 4$ (см. (7)). Пусть $T-$ неразложимое ортоскалярное представление размерности $\delta_{\widetilde{D}_{n}}$ соответствующего разделенного колчана $Q$. Для определенности будем считать, что $c_{1} \in \dot{Q}$.

Положим $A_{1}=T_{c_{1}, a_{1}}, A_{2}=T_{c_{1}, a_{2}}, X_{1}=T_{c_{1}, c_{2}}, X_{2}=T_{c_{3}, c_{2}}, \ldots, B_{1}=T_{c_{n-3}, b_{1}}$, $B_{2}=T_{c_{n-3}, b_{2}}$ при четном $n$ и $B_{1}=T_{b_{1}, c_{n-3}}, B_{2}=T_{b_{2}, c_{n-3}}$ при нечетном $n$.

Рассмотрим случай четного $n$. При $n=4$ ортоскалярные неразложимые представления колчана $Q$ в размерности $\delta_{\widetilde{D}_{4}}$ зависят (см. [7]) от $6=\left|Q_{v}\right|+1$ положительных параметров.

Пусть $n>4$. Допустимыми (унитарными) преобразованиями матриц представления матрицы $X_{1}, \ldots, X_{n-4}$ можно диагонализировать так, чтобы на диагоналях стояли неотрицательные числа. Пусть $A=\left[A_{1} \mid A_{2}\right], X_{i}=\left[\begin{array}{cc}x_{i} & 0 \\ 0 & y_{i}\end{array}\right]$, 
$i=1, \ldots, n-4, B=\left[B_{1} \mid B_{2}\right]$. Тогда из ортоскалярности представления $T$ следует, что матрицы $A A^{*}$ и $B B^{*}$ также диагональны. Пусть $A A^{*}=\left[\begin{array}{cc}x_{0}^{2} & 0 \\ 0 & y_{0}^{2}\end{array}\right], B B^{*}=$ $\left[\begin{array}{cc}x_{n-3}^{2} & 0 \\ 0 & y_{n-3}^{2}\end{array}\right]$. Тогда $\left[\begin{array}{cc}x_{i}^{2} & 0 \\ 0 & y_{i}^{2}\end{array}\right]+\left[\begin{array}{cc}x_{i+1}^{2} & 0 \\ 0 & y_{i+1}^{2}\end{array}\right]=\chi\left(c_{i+1}\right) I_{c_{i+1}}, i=0, \ldots, n-4$, и потому $x_{i}^{2}+x_{i+1}^{2}=y_{i}^{2}+y_{i+1}^{2}$, а значит,

$$
y_{i+1}^{2}=x_{i+1}^{2}+(-1)^{i}\left(x_{0}^{2}-y_{0}^{2}\right), \quad i=0, \ldots, n-4 .
$$

Если $x_{0}^{2}=y_{0}^{2}$ (либо $x_{j}^{2}=y_{j}^{2}$ для любого фиксированного $j$ ), то из (10) следует, что $x_{i}^{2}=y_{i}^{2}$ для $i=0, \ldots, n-3$. В этом случае матрица $\left[A_{1}\left|A_{2}\right| B_{1} \mid B_{2}\right]$ задает ортоскалярное представление $\widehat{T}$ колчана $\widehat{Q}$, соответствующего графу $\widetilde{D}_{4}$, и при переходе к эквивалентному представлению колчана $Q$, не меняющем приведенного вида матриц $X_{i}$, мы получаем переход к представлению колчана $\widehat{Q}$, эквивалентному представлению $\widehat{T}$. Такие представления колчана $\widehat{Q}$, как мы отметили выше, зависят не более чем от 6 параметров. Соответствующие представления колчана $Q$ (с учетом параметров $x_{1}, \ldots, x_{n-4}$ и соотношения $(10)$ ) зависят не более чем от $6+(n-4)=n+2=\left|Q_{v}\right|+1$ параметров.

Пусть $x_{i} \neq y_{i}$ при $i=0, \ldots, n-3$ и $\left(U_{i}\right)_{i \in G_{v}}$ осуществляют эквивалентность представления $T$ с ортоскалярным представлением $\widetilde{T}$, для которого $\widetilde{X}_{i}=X_{i}$, $i=0, \ldots, n-4\left(U_{i}\right.$ - унитарные матрицы). В этом случае легко убедиться, что $U_{i}=\left[\begin{array}{cc}u_{i} & 0 \\ 0 & v_{i}\end{array}\right]$ и в матрице $[A \mid B]=\left[\begin{array}{l|l|l|l}a_{11} & a_{12} & b_{11} & b_{12} \\ a_{21} & a_{22} & b_{21} & b_{22}\end{array}\right]$ можно все элементы, кроме двух, для определенности $b_{21}$ и $b_{22}$, сделать действительными и параметризовать следующим образом:

$$
[A \mid B]=\left[\begin{array}{c|c|c|c}
x_{0} \cos \phi_{1} & x_{0} \sin \phi_{1} & x_{n-3} \cos \phi_{2} & x_{n-3} \sin \phi_{2} \\
y_{0} \sin \phi_{1} & -y_{0} \cos \phi_{1} & y_{n-3} \sin \phi_{2} e^{i \theta} & -y_{n-3} \cos \phi_{2} e^{i \theta}
\end{array}\right] .
$$

Такие представления зависят от $n+2=\left|Q_{v}\right|+1$ положительных параметров $x_{0}, x_{1}, \ldots, x_{n-3}, y_{0}, \phi_{1}, \phi_{2}, \theta$.

Случай нечетного $n$ изучается аналогично с заменой матрицы $\left[A_{1}\left|A_{2}\right| B_{1} \mid B_{2}\right]$ на матрицу $\left[A_{1}\left|A_{2}\right| B_{1}^{*} \mid B_{2}^{*}\right]$

c) Пусть $G=\widetilde{E}_{6}$; соответствующий разделенный колчан $Q$ имеет вид

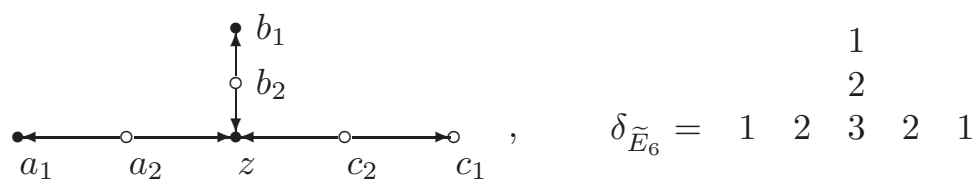

Пусть $T$ - неразложимое ортоскалярное представление колчана $Q$ в размерности $\delta_{\widetilde{E}_{6}}$,

$T=$\begin{tabular}{|c|c|c|}
\hline 0 & 0 & $C_{1}$ \\
\hline 0 & $B_{1}$ & 0 \\
\hline$A_{1}$ & 0 & 0 \\
\hline$A_{2}$ & $B_{2}$ & $C_{2}$ \\
\hline
\end{tabular}

$$
\begin{aligned}
D & =\left[\begin{array}{ll}
A_{2} & B_{2} C_{2}
\end{array}\right], & A_{2} & =\left[a_{i j}\right]_{j=1,2}^{i=1,2,3}, \\
B_{2} & =\left[b_{i j}\right]_{j=1,2}^{i=1,2,3}, & C_{2} & =\left[c_{i j}\right]_{j=1,2}^{i=1,2,3} .
\end{aligned}
$$

Здесь $A_{1}=T_{a_{1}, a_{2}}, B_{1}=T_{b_{1}, b_{2}}, C_{1}=T_{c_{1}, c_{2}}, A_{2}=T_{z, a_{2}}, B_{2}=T_{z, b_{2}}, C_{2}=T_{z, c_{2}}$. 
Унитарными преобразованиями столбцов приведем $A_{1}$ к виду $\left[0 x_{0}\right], x_{0}>0$. Унитарными преобразованиями строк матрицу $A_{2}$ приведем к виду
$a_{11}>0$. Из ортогональности столбцов в $\left.\begin{array}{cc}A_{11} & a_{12} \\ 0 & a_{22} \\ 0 & a_{32}\end{array}\right]$ следует, что $a_{12}=0$.

Унитарными преобразованиями столбцов (не меняя $A_{2}$ ) можно добиться, чтобы $b_{11}=0, c_{11}=0$; при этом $b_{12} \neq 0, c_{12} \neq 0-$ в противном случае можно проверить, что представление разложимо; допустимыми преобразованиями можно сделать $b_{12}$ и $c_{12}$ положительными. Унитарными преобразованиями двух последних строк матрицы $D$ можно сделать так, чтобы $b_{22}=0$, а тогда из ортогональности первых двух строк получим, что $c_{22}=0$. Итак,

$$
D=\left[\begin{array}{cc|cc|cc}
a_{11} & 0 & 0 & b_{12} & 0 & c_{12} \\
0 & a_{22} & b_{21} & 0 & c_{21} & 0 \\
0 & a_{32} & b_{31} & b_{32} & c_{31} & c_{32}
\end{array}\right]
$$

Значения характера $\chi(i)\left(i \in Q_{v}\right)$ связаны соотношением $\chi\left(a_{1}\right)+\chi\left(b_{1}\right)+$ $\chi\left(c_{1}\right)+3 \chi(z)=2\left(\chi\left(a_{2}\right)+\chi\left(b_{2}\right)+\chi\left(c_{2}\right)\right)$ (следует сумму квадратов модулей матричных элементов матрицы $T$ подсчитать двумя способами - сначала складывая эти квадраты модулей по строкам, а потом по столбцам). Перейдем к нормированному представлению с характером $\chi$, для которого $\chi(z)=1$, поделив все матрицы представления на одно и то же число $\sqrt{\chi(z)}$. Нормировка представления уменьшает минимальное числа параметров, от которых зависит представление, на единицу.

Вектор $\left(a_{11}, b_{12}, c_{12}\right)$, поскольку $a_{11}^{2}+b_{12}^{2}+c_{12}^{2}=1$, допускает параметризацию: $\left(a_{11}, b_{12}, c_{12}\right)=\left(\sin \psi_{1}, \sin \phi_{1} \cos \psi_{1}, \cos \phi_{1} \cos \psi_{1}\right)$. Так как первая и третья строки матрицы $D$ ортогональны, то $\left(b_{32}, c_{32}\right)=\left(\cos \phi_{1},-\sin \phi_{1}\right) x$, где $x \in \mathbb{C}$. Домножением третьей строки матрицы $D$ на число вида $e^{i \phi}$ (унитарное преобразование строк) сделаем элемент $x$ положительным, а потом аналогичным домножением пятого столбца на число сделаем элемент $c_{31}$ неотрицательным. Домножением второй строки матрицы $D$ на число сделаем элемент $c_{21}$ неотрицательным, а потом домножением второго и третьего столбцов - элементы $a_{22}$ и $b_{21}$ неотрицательными. Так как $a_{22}^{2}+b_{21}^{2}+c_{21}^{2}=1$, то вектор $\left(a_{22}, b_{21}, c_{21}\right)$ допускает параметризацию: $\left(a_{22}, b_{21}, c_{21}\right)=\left(\cos \phi_{2} \cos \psi_{2}, \sin \phi_{2} \cos \psi_{2}, \sin \psi_{2}\right)$. Элементы $a_{32}, b_{31}$ остаются, вообще говоря, комплексными. Пусть

$$
a_{32} \bar{a}_{32}+b_{31} \bar{b}_{31}+c_{31}^{2}=\sin ^{2} \psi_{3}, \quad b_{32} \bar{b}_{32}+c_{31}^{2}=\cos ^{2} \psi_{3} .
$$

Учитывая ортогональность первой и третьей строк матрицы $D$ и соотношения $(11)$, векторы $\left(b_{32}, c_{32}\right)$ и $\left(a_{32}, b_{31}, c_{31}\right)$ можно параметризовать так:

$$
\left(b_{32}, c_{32}\right)=\left(-\cos \phi_{1} \cos \psi_{3}, \sin \phi_{1} \cos \psi_{3}\right),
$$

$\left(a_{32}, b_{31}, c_{31}\right)=\left(\cos \phi_{3} \cos \psi_{4} \sin \psi_{3} e^{i \theta_{1}}, \sin \phi_{3} \cos \psi_{4} \sin \psi_{3} e^{i \theta_{2}}, \sin \psi_{4} \sin \psi_{3}\right)$.

Таким образом, матрица $D$, которую мы будем называть основой представления, зависит от 9 действительных параметров $\phi_{1}, \phi_{2}, \phi_{3}, \psi_{1}, \psi_{2}, \psi_{3}, \psi_{4}, \theta_{1}, \theta_{2}$. Из ортоскалярности представления следует ортонормированность строк в $D$. Ортогональность второй и третьей строк дает $\cos \phi_{2} \cos \phi_{3} \cos \psi_{2} \sin \psi_{3} \cos \psi_{4} e^{i \theta_{1}}$ $+\sin \phi_{2} \sin \phi_{3} \cos \psi_{2} \sin \psi_{3} \cos \psi_{4} e^{i \theta_{2}}+\sin \psi_{2} \sin \psi_{3} \sin \psi_{4}=0$, откуда получаем два 
«действительных» соотношения - равенство нулю действительной и мнимой части. Следовательно, из указанных параметров два можно исключить.

Пусть $A_{1}=\left[\begin{array}{ll}0 & x_{1}\end{array}\right], B_{1}=\left[b_{01} b_{02}\right], C_{1}=\left[\begin{array}{cc}c_{01} & c_{02}\end{array}\right]$. Унитарными преобразованиями строк элементы $b_{01}$ и $c_{01}$ можно сделать действительными неотрицательными - пусть $b_{01}=y_{0}, b_{02}=y_{1} e^{i \theta}$.

Покажем, что тогда основой $D$ ортоскалярное представление $T$ определяется однозначно.

Из условий ортоскалярности вытекает, что $x_{0}^{2}+a_{22}^{2}+a_{32} \bar{a}_{32}=a_{11}^{2}$, так что $x_{0}$ по основе представления определяется однозначно. Также имеем $b_{01}^{2}+b_{21}^{2}+$ $\left|b_{31}\right|^{2}=\left|b_{02}\right|^{2}+b_{12}^{2}+b_{32}^{2}$, так что $b_{01}^{2}-\left|b_{02}\right|^{2}=b_{12}^{2}+b_{32}^{2}-b_{21}^{2}-\left|b_{31}\right|^{2}=s$, и $b_{01} b_{02}+\overline{b_{31}} b_{32}=0$, так что $b_{01} b_{02}=b_{01}\left|b_{02}\right| e^{i \theta}=-\left|b_{31}\right| e^{i \theta_{2}} b_{32}$. Поэтому $e^{i \theta}=$ $-e^{i \theta_{2}}$ и $b_{01}^{2}\left(-\left|b_{02}\right|^{2}\right)=-\left|b_{31}\right| b_{32}=-t$, где $t>0$. Стало быть, числа $b_{01}^{2}$ и $-\left|b_{02}\right|^{2}$ суть корни квадратного уравнения $z^{2}-s \cdot z-t=0, t>0$. Уравнение имеет два действительных корня разного знака; таким образом, $b_{01}$ и $b_{02}$ определяются основой представления однозначно.

Аналогично по основе представления однозначно определяются и числа $c_{01}$, $c_{02}$. Стало быть, в размерности $\delta_{\widetilde{E}_{6}}$ неразложимые нормированные ортоскалярные представления зависят от 7 независимых параметров, а неразложимые (не обязательно нормированные) - от $8=\left|Q_{v}\right|+1$ независимых параметров.

d) Пусть $G=\widetilde{E}_{7}$; соответствующий разделенный колчан $Q$ имеет вид

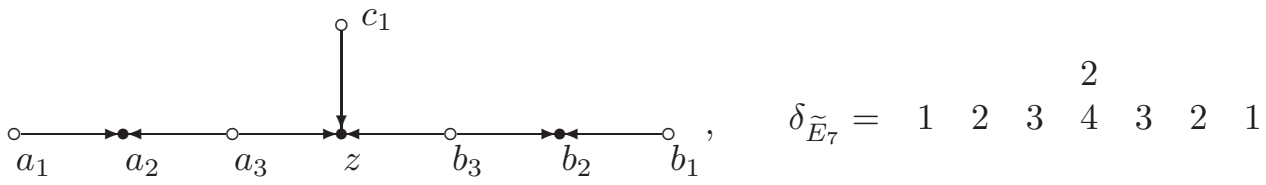

$T$ - неразложимое ортоскалярное представление колчана $Q$ в размерности $\delta_{\widetilde{E}_{7}}$. $T=\left[A_{i j}\right]_{j=1, \ldots, 5}^{i=1,2,3}=\left[a_{i j}\right]_{j=1, \ldots, 10}^{i=1, \ldots, 8}, A_{11}=T_{a_{2}, a_{1}}, A_{12}=T_{a_{2}, a_{3}}, A_{22}=T_{z, a_{3}}, A_{23}=$ $T_{z, c_{1}}, A_{24}=T_{z, b_{3}}, A_{34}=T_{b_{2}, b_{3}}, A_{35}=T_{b_{2}, b_{1}}$, остальные блоки нулевые.

Допустимыми унитарными преобразованиями строк и столбцов некоторые из элементов матрицы $T$ преобразуем в нули, некоторые из элементов станут равными нулю в силу ортогональности строк и столбцов внутри полос.

Символ $\overline{0}_{k}$ на каком-либо место матрицы $T$ будет означать, что на этом месте может быть получен нуль на $k$-м шаге унитарными преобразованиями столбцов вертикальной полосы, $\left.0\right|_{k}-$ строк горизонтальной полосы, $\overrightarrow{0}_{k}-$ нуль получен в силу ортогональности столбцов вертикальной полосы, $0 \downarrow_{k}$ - в силу ортогональности строк. При этом, делая нули на $k$-м шаге, мы не «портим» нули, полученные ранее. Умножением строк и столбцов на числа вида $e^{i \phi}$ (унитарные преобразования) некоторые из элементов можно сделать действительными неотрицательными, и мы на это укажем прямо в матрице $\left(a_{i j} \geqslant 0\right.$ либо $\left.a_{i j} \leqslant 0\right)$. Мы указываем на строгое сравнение $\left(a_{i j}>0\right.$ либо $\left.a_{i j}<0\right)$, если из равенства элемента $a_{i j}$ нулю следует разложимость представления. В результате мы получим следующие значения элементов матрицы $T: a_{11} \geqslant 0, a_{12}=\left.0\right|_{11}, a_{13} \geqslant 0$, $a_{14} \leqslant 0, a_{21} \geqslant 0, a_{22}>0, a_{23} \leqslant 0, a_{24}=\overrightarrow{0}_{12}, a_{32}>0, a_{33}=\overline{0}_{2}, a_{34}=\overline{0}_{2}$, $a_{35}>0, a_{36}=\overline{0}_{2}, a_{37}=\left.\overline{0}\right|_{1}, a_{38}=\left.\overline{0}\right|_{1}, a_{39}>0, a_{42}>0, a_{43} \geqslant 0, a_{44}=\overline{0}_{6}$, $a_{45}<0, a_{46}=\overrightarrow{0}_{5}, a_{47}>0, a_{48}=\overline{0}_{6}, a_{49}=\left.\overline{0}\right|_{1}, a_{52}=\left.0\right|_{3}, a_{53} \geqslant 0, a_{54} \geqslant 0$, 
$a_{55}=0 \downarrow_{4}, a_{56} \geqslant 0, a_{57} \leqslant 0, a_{58} \geqslant 0, a_{59}=\left.\overline{0}\right|_{1}, a_{62}=\left.0\right|_{3}, a_{63}=\left.0\right|_{7}, a_{64} \geqslant 0$, $a_{65}=0 \downarrow_{4}, a_{66} \in \mathbb{C}, a_{67}=0 \downarrow_{8}, a_{68} \in \mathbb{C}, a_{69}=\left.\overline{0}\right|_{1}, a_{77}=\left.0\right|_{9}, a_{78} \geqslant 0, a_{79}=\overrightarrow{0}_{10}$, $a_{7,10} \geqslant 0, a_{87} \geqslant 0, a_{88} \geqslant 0, a_{89}=\overrightarrow{0}_{9}, a_{8,10} \leqslant 0$. Остальные элементы изначально были равны 0 . Нормируем представление $T$ (будем считать, что $\chi(z)=1$ ). Тогда матрицу $D=\left[\begin{array}{lll}A_{22} & A_{23} & A_{24}\end{array}\right]$ (будем называть ее основой представления) можно параметризовать следующим образом:

$$
A_{22}=\left[\begin{array}{ccc}
\cos \phi_{1} \cos \psi_{1} & 0 & 0 \\
\sin \phi_{1} \cos \psi_{2} & \cos \phi_{2} \sin \psi_{2} & 0 \\
0 & \sin \phi_{2} \cos \psi_{4} & \cos \phi_{3} \cos \psi_{3} \sin \psi_{4} \\
0 & 0 & \sin \phi_{4} \sin \psi_{5}
\end{array}\right]
$$

$\left[A_{23} \mid A_{24}\right]=\left[\begin{array}{cc|ccc}\sin \phi_{1} \cos \psi_{1} & 0 & 0 & 0 & \sin \psi_{1} \\ -\cos \phi_{1} \cos \psi_{2} & 0 & \sin \phi_{2} \sin \psi_{2} & 0 & 0 \\ 0 & \sin \phi_{3} \cos \psi_{3} \sin \psi_{4} & -\cos \phi_{2} \cos \psi_{4} & \sin \psi_{3} \sin \phi_{4} & 0 \\ 0 & \cos \phi_{4} \sin \psi_{5} e^{i \theta_{1}} & 0 & \cos \psi_{5} e^{i \theta_{2}} & 0\end{array}\right]$.

Из ортогональности третьей и четвертой строк матрицы $D$ следует, что

$\cos \phi_{3} \sin \phi_{4} \cos \psi_{3} \sin \psi_{4} \sin \psi_{5}$

$+\sin \phi_{3} \cos \phi_{4} \cos \psi_{3} \sin \psi_{4} \sin \psi_{5} e^{i \theta_{1}}+\sin \psi_{3} \sin \psi_{4} \cos \psi_{5} e^{i \theta_{2}}=0$.

Это соотношение эквивалентно двум действительным. Из равенства длин столбцов матрицы $A_{23}$ следует, что

$$
\sin ^{2} \phi_{2} \cos ^{2} \psi_{1}+\cos ^{2} \phi_{1} \cos ^{2} \psi_{2}=\sin ^{2} \phi_{3} \cos ^{2} \psi_{3} \sin ^{2} \psi_{4}+\cos ^{2} \phi_{4} \sin ^{2} \psi_{5},
$$

так что из 11 параметров $\phi_{1}-\phi_{4}, \psi_{1}-\psi_{5}, \theta_{1}, \theta_{2}$ независимыми являются 8 .

Легко видеть, что по основе $D$ представления $T$ матрицы $A_{11}, A_{12}, A_{34}, A_{35}$ (ранее частично приведенные) находятся однозначно.

Так, из ортоскалярности столбцов матрицы $\left[\begin{array}{l}A_{24} \\ A_{34}\end{array}\right]$ следует, что

$$
a_{87}^{2}+a_{47}^{2}+a_{54}^{2}=a_{39}^{2}, \quad a_{87} a_{88}+a_{54} a_{58}=0, \quad a_{78}^{2}+a_{88}^{2}+a_{58}^{2}+a_{68} \overline{a_{68}}=a_{39}^{2} .
$$

Отсюда последовательно и однозначно находятся элементы $a_{87}, a_{88}, a_{78}$. Из ортоскалярности строк матрицы $\left[\begin{array}{ll}A_{34} & A_{35}\end{array}\right]$ вытекает, что $a_{78}^{2}+a_{7,10}^{2}=a_{87}^{2}+$ $a_{88}^{2}+a_{8,10}^{2}, a_{78} a_{88}+a_{7,10} a_{8,10}=0$, откуда находятся элементы $a_{7,10}$ и $a_{8,10}$.

Параметризуем столбцы матрицы $\left[\begin{array}{l}A_{12} \\ A_{22}\end{array}\right]$ следующим образом:

$$
\left[\begin{array}{l}
A_{12} \\
A_{22}
\end{array}\right]=\left[\begin{array}{ccc}
0 & x \sin \alpha_{2} \sin \beta_{2} & -x \sin \beta_{3} \\
x \sin \beta_{1} & -x \cos \alpha_{2} \sin \beta_{2} & 0 \\
\hline x \cos \alpha_{1} \cos \beta_{1} & 0 & 0 \\
x \sin \alpha_{1} \cos \beta_{1} & x \sin \alpha_{3} \cos \beta_{2} & 0 \\
0 & x \cos \alpha_{3} \cos \beta_{2} & x \sin \alpha_{4} \cos \beta_{3} \\
0 & 0 & x \cos \alpha_{4} \cos \beta_{3}
\end{array}\right] ;
$$

здесь $x>0$.

Покажем, что элементы матрицы $A_{12}$ элементами матрицы $A_{22}$ определяются однозначно. Так как $x \cos \alpha_{1} \cos \beta_{1}=a_{32}, x \sin \alpha_{1} \cos \beta_{1}=a_{42}$, то $x^{2} \cos ^{2} \beta_{1}=$ 
$a_{32}^{2}+a_{42}^{2}$. Аналогично, $x^{2} \cos ^{2} \beta_{2}=a_{43}^{2}+a_{53}^{2}$ и $x^{2} \cos ^{2} \beta_{3}=a_{54}^{2}+a_{64}^{2}$. Следовательно, $x \sin \beta_{1}=\sqrt{x^{2}-\left(a_{32}^{2}+a_{42}^{2}\right)}, x \sin \beta_{2}=\sqrt{x^{2}-\left(a_{43}^{2}+a_{53}^{2}\right)}, x \sin \beta_{3}=$ $\sqrt{x^{2}-\left(a_{54}^{2}+a_{64}^{2}\right)}$. Из ортогональности столбцов матрицы $\left[\begin{array}{l}A_{12} \\ A_{22}\end{array}\right]$ следует, что

$-x^{2} \sin \beta_{1} \sin \beta_{2} \cos \alpha_{2}+a_{42} a_{43}=0$, $-x^{2} \sin \beta_{2} \sin \beta_{3} \sin \alpha_{2}+a_{53} a_{54}=0 ;$

из этих равенств находятся $\cos \alpha_{2}$ и $\sin \alpha_{2}$.

Значение $x=\chi\left(a_{3}\right)$ определяется по основе представления однозначно: если $A_{22}$ унитарно эквивалентна матрице $\operatorname{diag}\left\{r_{1}, r_{2}, r_{3}\right\}, r_{i} \geqslant 0$, то $x=\max _{i} r_{i}$ (это легко получить, диагонализируя матрицы $A_{12}, A_{22}$ унитарными преобразованиями, при этом существенно используется ортоскалярность представления). Элементы $a_{11}, a_{21}$ однозначно находятся по матрице $A_{12}$ (аналогично тому, как находятся элементы $a_{7,10}, a_{8,10}$ по матрице $\left.A_{34}\right)$.

Стало быть, (ненормированные) неразложимые ортоскалярные представления в размерности $\delta_{\tilde{E}_{8}}$ зависят от $9=\left|Q_{v}\right|+1$ независимых параметров.

е) Пусть $G=\widetilde{E}_{8} ;$ соответствующий разделенный колчан имеет вид

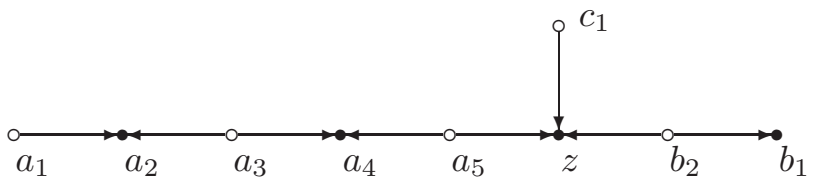

3

Как известно, $\delta_{\widetilde{E}_{8}}=\begin{array}{lllllllll}1 & 2 & 3 & 4 & 5 & 6 & 4 & 2\end{array}$.

Пусть $T$ - неразложимое ортоскалярное представление колчана $Q$ в размерности $\delta_{\widetilde{E}_{8}}, T=\left[A_{i j}\right]_{j=1, \ldots, 5}^{i=1, \ldots, 4}=\left[a_{i j}\right]_{j=1, \ldots, 16}^{i=1, \ldots, 14}$, где $A_{11}=T_{a_{2}, a_{1}}, A_{12}=T_{a_{2}, a_{3}}$, $A_{22}=T_{a_{4}, a_{3}}, A_{23}=T_{a_{4}, a_{5}}, A_{33}=T_{z, a_{5}}, A_{34}=T_{z, c_{1}}, A_{35}=T_{z, b_{2}}, A_{45}=T_{b_{1}, b_{2}}$; остальные блоки равны нулю. Следуя предыдущим договоренностям об обозначениях, матрицу $T$ можно привести к виду, при котором $a_{11}>0, a_{12}>0$, $a_{13}=\overrightarrow{0}_{26}, a_{14}=\overrightarrow{0}_{26}, a_{21}=0 \downarrow_{27}, a_{22}=\left.0\right|_{25}, a_{23}>0, a_{24}<0, a_{32}>0, a_{33}=\overline{0}_{21}$, $a_{34}=\overline{0}_{21}, a_{35}>0, a_{36}=\overrightarrow{0}_{17}, a_{37}=\overrightarrow{0}_{17}, a_{38}=\overrightarrow{0}_{17}, a_{39}=\overrightarrow{0}_{17}, a_{42}=0 \downarrow_{22}$, $a_{43}>0, a_{44}=\overline{0}_{23}, a_{45}=\left.0\right|_{16}, a_{46}>0, a_{47}<0, a_{48}=\overrightarrow{0}_{19}, a_{49}=\overrightarrow{0}_{19}, a_{52}=0 \downarrow_{22}$, $a_{53}>0, a_{54}>0, a_{55}=\left.0\right|_{16}, a_{56}=\left.0\right|_{18}, a_{57}>0, a_{58}<0, a_{59}=\overrightarrow{0}_{21}, a_{62}=0 \downarrow_{22}$, $a_{63}=0 \downarrow_{24}, a_{64}>0, a_{65}=\left.0\right|_{16}, a_{66}=\left.0\right|_{18}, a_{67}=\left.0\right|_{20}, a_{68}>0, a_{69}<0, a_{75}>0$, $a_{76}=\left.\overline{0}\right|_{1}, a_{77}=\left.\overline{0}\right|_{1}, a_{78}=\left.\overline{0}\right|_{1}, a_{79}=\left.\overline{0}\right|_{1}, a_{7,10}>0, a_{7,11}=\overline{0}_{2}, a_{7,12}=\overline{0}_{2}$, $a_{7,13}>0, a_{7,14}=\overline{0}_{2}, a_{7,15}=\overline{0}_{2}, a_{7,16}=\overline{0}_{2}, a_{85}=\left.\overline{0}\right|_{1}, a_{86} \geqslant 0, a_{87}=\overline{0}_{6}, a_{88}=\overline{0}_{6}$, $a_{89}=\overline{0}_{6}, a_{8,10}>0, a_{8,11}=\overrightarrow{0}_{6}, a_{8,12}=\overrightarrow{0}_{6}, a_{8,13}<0, a_{8,14}>0, a_{8,15}=\overrightarrow{0}_{6}$, $a_{8,16}=\overrightarrow{0}_{6}, a_{95}=\left.\overline{0}\right|_{1}, a_{96} \geqslant 0, a_{97} \geqslant 0, a_{98}=\overline{0}_{7}, a_{99}=\overline{0}_{7}, a_{9,10}=\left.0\right|_{3}, a_{9,11}>0$, $a_{9,12}=\overline{0}_{7}, a_{9,13}=0 \downarrow_{4}, a_{9,14}<0, a_{9,15}=\overrightarrow{0}_{9}, a_{9,16}=\overrightarrow{0}_{9}, a_{10,5}=\left.\overline{0}\right|_{1}, a_{10,6}=\left.0\right|_{7}$, $a_{10,7} \geqslant 0, a_{10,8} \geqslant 0, a_{10,9}=\overline{0}_{11}, a_{10,10}=\left.0\right|_{3}, a_{10,11}<0, a_{10,12}=\overline{0}_{13}, a_{10,13}=0 \downarrow_{4}$, $a_{10,14}=0 \downarrow_{8}, a_{10,15}>0, a_{10,16}=\overline{0}_{14}, a_{11,5}=\left.\overline{0}\right|_{1}, a_{11,6}=\left.0\right|_{7}, a_{11,7}=\left.0\right|_{10}, a_{11,8} \geqslant 0$, $a_{11,9} \geqslant 0, a_{11,10}=\left.0\right|_{3}, a_{11,11}=0 \downarrow_{14}, a_{11,12} \geqslant 0, a_{11,13}=0 \downarrow_{4}, a_{11,14}=0 \downarrow_{8}$, $a_{11,15}<0, a_{11,16} \geqslant 0, a_{12,5}=\left.\overline{0}\right|_{1}, a_{12,6}=\left.0\right|_{7}, a_{12,7}=\left.0\right|_{10}, a_{12,8}=0, a_{12,9} \geqslant 0$, $a_{12,10}=\left.0\right|_{3}, a_{12,11}=0 \downarrow_{14}, a_{12,12} \in \mathbb{C}, a_{12,13}=0 \downarrow_{4}, a_{12,14}=0 \downarrow_{8}, a_{12,15}=0 \downarrow_{15}$, 
$a_{12,16} \in \mathbb{C}, a_{13,13}>0, a_{13,14}>0, a_{13,15}=\overline{0}_{5}, a_{13,16}=\overline{0}_{5}, a_{14,13}=\left.0\right|_{5}, a_{14,14}=0 \downarrow_{5}$, $a_{14,15}>0, a_{14,16}>0$.

Назовем основой представления матрицу $D=\left[\begin{array}{ccc}A_{33} & A_{34} & A_{35} \\ 0 & 0 & A_{45}\end{array}\right]$. Ее можно параметризовать следующим образом: $a_{75}=\sin \psi_{1}, a_{7,10}=\cos \phi_{1} \cos \psi_{1}, a_{7,13}=$ $\sin \phi_{1} \cos \psi_{1}, a_{86}=\sin \phi_{2} \sin \psi_{2}, a_{8,10}=\sin \phi_{1} \cos \psi_{2}, a_{8,13}=-\cos \phi_{1} \cos \psi_{2}$, $a_{8,14}=\cos \phi_{2} \sin \psi_{2}, a_{96}=\cos \phi_{2} \sin \psi_{3}, a_{97}=\sin \phi_{3} \cos \psi_{3}, a_{9,11}=\cos \phi_{3} \cos \psi_{3}$, $a_{9,14}=-\sin \phi_{2} \sin \psi_{3}, a_{10,7}=\cos \phi_{3} \sin \psi_{4}, a_{10,8}=\sin \phi_{4} \cos \psi_{4}, a_{10,11}=$ $-\sin \phi_{3} \sin \psi_{4}, a_{10,15}=\cos \phi_{4} \cos \psi_{4}, a_{11,8}=\cos \phi_{4} \cos \psi_{5}, a_{11,9}=\cos \phi_{5} \sin \psi_{5} \cos \psi_{6}$, $a_{11,12}=\sin \phi_{5} \sin \psi_{5} \cos \psi_{6}, a_{11,15}=-\sin \phi_{4} \cos \psi_{5}, a_{11,16}=\sin \psi_{5} \sin \psi_{6}, a_{12,9}=$ $\cos \phi_{6} \cos \psi_{7}, a_{12,12}=\sin \phi_{6} \cos \psi_{7} e^{i \theta_{1}}, a_{12,16}=-\sin \psi_{7} e^{i \theta_{2}}, a_{13,13}=r \cos \phi_{7}$, $a_{13,14}=r \sin \phi_{7}, a_{14,15}=r \cos \phi_{8}, a_{14,16}=r \sin \phi_{8}$.

Так же, как и в предыдущих случаях, можно показать, что по основе $D$ представления $T$ матрицы $A_{11}, A_{12}, A_{22}, A_{23}$ (ранее частично приведенные) находятся однозначно.

При выбранной параметризации многие соотношения, обеспечивающие ортоскалярность, уже выполняются. Дополнительно для элементов основы $D$ следует потребовать ортогональность пятой и шестой строк (2 действительных соотношения), первого столбца со вторым, а также третьего с четвертым (еще 2 соотношения), равенство «длин» столбцов матрицы $\left[\begin{array}{l}A_{35} \\ A_{45}\end{array}\right]$ (3 соотношения). Таким образом, 18 параметров $\phi_{1}-\phi_{8}, \psi_{1}-\psi_{7}, \theta_{1}, \theta_{2}$ и $r$ нормированного представления связаны 9 соотношениями и зависят от 9 независимых параметров, а ненормированные неразложимые ортоскалярные представления в размерности $\delta_{\tilde{E}_{8}}$ зависят от $10=\left|Q_{v}\right|+1$ независимых параметров.

Замечание 2. Как и для категории линейных пространств, размерности неразложимых ортоскалярных представлений расширенных графов Дынкина не ограничены в совокупности. В размерности, являющейся действительным корнем графа, при фиксированном характере неразложимое представление единственно. Для размерностей, являющихся мнимыми корнями графа, неразложимые ортоскалярные представления, в отличие от линейно-алгебраического случая, существуют только в размерности, совпадающей с минимальным положительным мнимым корнем, но также зависят при фиксированном характере, от одного (комплексного) параметра.

\section{ЛиТЕРАТУРА}

[1] A. V. Roiter, Matrix problems, in: Proc. ICM Helsinki, 1978, Acad. Sci. Fennica, Helsinki, 1980, 319-322.

[2] С. А. Кругляк, А. В. Ройтер, Локально-скалярные представления графов в категории гилъбертовых пространств, Функц. анализ и его прил., 39:2 (2005), 13-30.

[3] P. Gabriel, Unzerlegbare Darstellungen I, Manuscripta Math., 6 (1972), 71-107.

[4] С. А. Кругляк, В. И. Рабанович, Ю. С. Самойленко, О суммах проекторов, Функц. анализ и его прил., 36:3 (2002), 20-35.

[5] В. Л. Островський, Ю. С. Самойленко, Про спектральні теореми для сімей лінійно пов'язаних самосопряжених операторів із заданими спектрами, що асоиійовані з розширеними графами Динкіна, Укр. матем. ж., 58:11 (2006), 1556-1570.

[6] S. Albeverio, V. Ostrovskyi, Yu. Samoilenko, On functions on graphs and representations of a certain class of $*$-algebras, J. Algebra, 308:2 (2007), 567-582. 
[7] С. А. Кругляк, Л. А. Назарова, А. В. Ройтер, Ортоскалярные представления колчанов в категории гильбертовых пространств, Зап. научн. сем. ПОМИ, 338 (2006), 180-199.

[8] А. В. Ройтер, Боксы с инволючией, в кн.: Представления и квадратичные формы, Ин-т математики АН УССР, Киев, 1979, 124-126, 155.

[9] Ж. Диксмье, $C^{*}$-алгебры и их представления, Наука, М., 1974.

[10] V. G. Kac, Infinite root systems, representations of graphs and invariant theory, II, J. Algebra, 78 (1982), 141-162.

[11] I. M. Gelfand, V. A. Ponomarev, Problems of linear algebra and classification of quadruples of subspaces in a finite-dimensional vector space, in: Hilbert Space Operators and Operator Algebra (Proc. Intern. Conf., Tihany, Hungary, 1970), Colloq. Math. Soc. J. Bolyai, vol. 5, 1972, 163-237.

[12] И. К. Редчук, А. В. Ройтер, Сингулярные локально-скалярные представления колчанов в гильбертовых пространствах и разделяющие функиии, Укр. матем. ж., 56:6 (2004), 796-809.

[13] W. Crawley-Boevey, Lectures on Representations of Quivers, Preprojective Algebras and Deformations of Quotient Singularities, http://www.maths.leeds.ac.uk/ pmtwc/dmvlecs.pdf.

[14] V. L. Ostrovskyi, Yu. S. Samoilenko, Introduction to the theory of representations of finitely presented *-algebras, I, Rev. Math. Math. Phys., 11 (1999), 1-261.

[15] А. С. Меллит, Когда сумма трех частичных отражсений равна нулю, Укр. матем. ж., 55:9 (2003), 1277-1283.

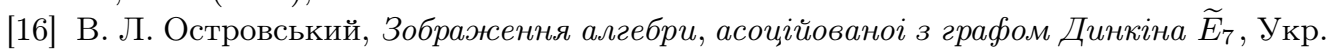
матем. ж., 56:9 (2004), 1193-1204.

Институт математики НАН Украины

e-mail: krug@ehl.kiev.ua

Поступило в редакцию

Институт математики НАН Украины 13 февраля 2009 г.

e-mail: nazarovala@yahoo.co.uk

Институт математики НАН Украины 